\title{
Protective effect of thymoquinone on cholestatic rats with liver injury
}

\author{
L.Y. Kong', G.P. Li ${ }^{1}$, P. Yang ${ }^{1}$ and Z. Xi ${ }^{2}$ \\ ${ }^{1}$ Department of Emergency, \\ The First Affiliated Hospital of Xinxiang Medical University, \\ Weihui, Henan, China \\ ${ }^{2}$ Department of Pediatric Surgery, \\ The First Affiliated Hospital of Xinxiang Medical University, \\ Weihui, Henan, China \\ Corresponding author: Z. Xi \\ E-mail: lydoccn@126.com
}

Genet. Mol. Res. 14 (4): 12247-12253 (2015)

Received March 4, 2015

Accepted July 8, 2015

Published October 9, 2015

DOI http://dx.doi.org/10.4238/2015.October.9.13

ABSTRACT. The aim of this study was to investigate the protective
effects of thymoquinone treatment on cholestatic rats with liver injury.
Thirty-two Sprague-Dawley rats were divided randomly into four
groups: normal control, bile duct ligation model control, low-dose
thymoquinone ( $25 \mathrm{mg} / \mathrm{kg})$, and high-dose thymoquinone ( $50 \mathrm{mg} / \mathrm{kg}$ ).
Thymoquinone gavage was administered continuously 3 days before
bile duct ligation, and saline, at the same volume, was administered to the
control group. The rats were sacrificed after 2 weeks of treatment, and the
liver tissues were obtained and frozen. The contents of hydroxyproline
(HP), malondialdehyde (MDA), superoxide dismutase (SOD), and
glutathione peroxidase (GPx) in the homogenate of the liver tissues
were determined to evaluate the changes in hepatic tissue pathology by
fibrosis scoring. The HP and MDA levels were significantly lower and
the SOD and GPx levels were significantly higher in the thymoquinone-
treatment group than the corresponding levels in the model control 
group, and the differences were statistically significant $(\mathrm{P}<0.05)$ and dose-dependent. The hepatic necrosis areas and hepatic fibrosis scores of the thymoquinone-treatment groups were significantly lower than those of the model group $(\mathrm{P}<0.05)$. Thymoquinone increased the antioxidative capacity of liver and reduced the oxidative stress damage to the liver. Thymoquinone can be used as a liver protectant in patients with cholestasis.

Key words: Thymoquinone; Cholestasis; Liver protectant

\section{INTRODUCTION}

Choletastis is the disruption of bile duct excretion due to obstables at the bile duct or liver damage. Cholestasis is the main pathological feature of chronic liver diseases, such as biliary atresia, primary biliary cirrhosis, and primary sclerosing cholangitis (Figiel et al., 2012), and is one of the underlying causes of liver cell damage, liver fibrosis, cirrhosis, and fatal liver failure (Tomur et al., 2011). Existing studies have not been able to clarify fully the mechanism of liver injury caused by cholestasis. However, some studies have shown that free radicals, oxidative stress, and lipid peroxidation have important functions in the course of cholestasis (El-Mahmoudy et al., 2002; Copple et al., 2010). Therefore, oxidative damage serves as an important starting point in the research and treatment of cholestasis. Rat models with ligated bile ducts show the following symptoms, which are similar to those observed in humans with biliary hepatic fibrosis: duct occlusion causing lipid peroxidation, increased necrosis and apoptosis of liver cells, activated hepatic stellate cells, proliferation of bile duct epithelial cells, promotion of remodeling hepatic fibrous scaffold, and fibrosis development (Tomur et al., 2011; Galicia-Moreno et al., 2012). Immunohistochemical study on bile duct ligation models revealed hepatic fibrosis lesions containing a large number of $\alpha$-smooth muscle actin-positive cells; these cells might have originated from fat storage cells or myofibroblasts, which play a key role in the pathogenesis of liver fibrosis (Mansour, 2000; Kanter et al., 2005).

Previous studies showed that thymoquinone, one of the drugs extracted from black grass seed, has various biological activities and has been used widely in the treatment of asthma, eczema, high blood pressure, and other diseases. Recent studies reveal that thymoquinone helps inhibit the production of arachidonic acid and that it has anti-lipid peroxidation and anti-tumor effects (Butler et al., 2010; Singer et al., 2011). Information regarding the protective effect of thymoquinone and its mechanism of action in cholestatic liver cells is lacking. In the present study, the antioxidant and protective effects of thymoquinone on liver function and stress injury were determined by investigating the activities of hydroxyproline (HP), malondialdehyde (MDA), superoxide dismutase (SOD), and glutathione peroxidase (GPx) in the liver tissues of bile duct ligation rat models.

\section{MATERIAL AND METHODS}

\section{Animals and breeding condition}

Specific pathogen-free, male Sprague-Dawley (SD) rats (aged 16 weeks old; weigh- 
ing 200 to $220 \mathrm{~g}$ ) used in the experiment were provided by the Experimental Animal Center of Henan Province, China, with experimental animal certificate No. SYXK 00015882. The rearing conditions simulated normal light, temperature, and humidity. Animals were provided free access to food and water and were reared in an alternating 12-h light/12-h dark photoperiod and relative humidity of 55 to $60 \%$. This study was carried out in strict accordance with the recommendations in the Guide for the Care and Use of Laboratory Animals of the National Institutes of Health. The animal use protocol has been reviewed and approved by the Institutional Animal Care and Use Committee (IACUC) of Xinxiang Medical University.

\section{Grouping and treatment}

Thirty-two male SD rats were divided randomly into four groups: normal control, normal model, low-dose thymoquinone, and high-dose thymoquinone. Eight rats were assigned to each group. Thymoquinone (Sigma-Aldrich Corp., St. Louis, MO, USA) was dissolved in dimethylsulfoxide (DMSO) solution prior to experimentation and diluted with normal saline to a final DMSO concentration of $<0.5 \%$. Thymoquinone at 25 and $50 \mathrm{mg} / \mathrm{kg}$ was administered continuously to the low-dose and high-dose thymoquinone groups, respectively, for 3 consecutive days. The other groups were treated with commensurable normal saline and DMSO for the same period. Except for those in the normal control group, urethane solution (Shanghai Research Extension Biological Technology Co., Shanghai, China) was injected intraperitoneally to the animals at a dose of $1.2 \mathrm{~g} / \mathrm{kg}$ as anesthesia. Rats were supinely fixed, and median laparotomy was performed to expose the duct. The duct was ligated with $5 / 0$ cable to prepare the extrahepatic cholestasis model. Thymoquinone was administered continuously to the treatment groups, and commensurable normal saline and DMSO were administered to the other animals. Animals were sacrificed after 2 weeks of treatment. The livers were obtained and frozen for preservation in liquid nitrogen. Biochemical tests and histopathological evaluation were performed.

\section{Biochemical parameter detection}

Full homogenates of liver tissue $(10 \%)$ were prepared and centrifuged at low speed (Hunan Instrument Centrifuge Factory, Changsha, China) at $1409 \mathrm{~g}$ for 10 min to obtain the supernatant. The contents of HP, MDA, SOD, and GPx were detected using the HFY36-HF240 automatic biochemical analyzer (Tylenol Electronics Co., Ltd., Hangzhou, China) according to the manufacturer instructions.

\section{Morphological analysis}

The tissues from the right liver lobe of rats were obtained and embedded into paraffin. Sections ( $4 \mu \mathrm{m}$ thick) were prepared, dewaxed, stained with hematoxylin for $5 \mathrm{~min}$, and rinsed with water. Sections were treated with hydrochloric acid/ethanol for $30 \mathrm{~s}$ for differentiation and then soaked in clean water for $15 \mathrm{~min}$. Sections were stained with eosin for $2 \mathrm{~min}$, dehydrated, and then mounted using neutral gum. The portal and lobular areas were observed under a BX41 biological microscope (OLYMPUS Corp., Tokyo, Japan). Based on the scores 
obtained using the Ishak Standards, liver pathology was divided into four degrees: 0 (-), normal liver tissue with no obvious degeneration, necrosis, and inflammatory cell infiltration; 1 $(+)$, normal hepatic lobules with apparent cloudy swelling, ballooning degeneration or fatty degeneration, and scattered punctate necrosis; $2(++)$, unclear lobular structure and obvious focal necrosis with inflammatory cell infiltration; $3(+++)$, unclear lobular structure and obvious necrosis associated with inflammatory cell infiltration.

\section{Statistical analysis}

Data are reported as means \pm standard deviation and were analyzed by the SPSS v17.0 statistical software (SPSS Inc., USA). Comparisons among multiple groups were performed using one-way ANOVA. $\mathrm{P}<0.05$ was considered to be statistically significant.

\section{RESULTS}

\section{Clinical symptoms}

After bile duct ligation, the SD rats started showing symptoms of cholestasis, such as messy and dull hair, reduced food consumption, slow activity, depression, powdery white feces, and yellow urine. Rats in the thymoquinone-treatment groups showed better mental state and better food intake than those in the model control groups. Compared to the rats in the model groups, rats in the treatment groups had relatively normal hair and the color changes in feces and urine were less.

\section{Liver biochemical parameters}

As shown in Table 1, the HP, MDA, SOD, and GPx levels in the normal control, model control, low-dose treatment, and high-dose treatment groups were $226.13 \pm 12.56$, $568.92 \pm 20.18,369.58 \pm 18.39$, and $282.47 \pm 9.59 \mathrm{mg} / \mathrm{g} ; 186.81 \pm 10.05,597.57 \pm 22.31$, $324.18 \pm 16.4$, and $269.77 \pm 18.6 \mathrm{nmol} / \mathrm{g} ; 5.34 \pm 0.82,2.15 \pm 0.25,3.46 \pm 0.33$, and $4.12 \pm 0.38 \mathrm{U} / \mathrm{g}$; and $0.56 \pm 0.09,0.25 \pm 0.04,0.46 \pm 0.04$, and $0.49 \pm 0.06 \mathrm{nmol} / \mathrm{g}$, respectively. The HP and MDA levels were significantly lower and the SOD and GPX levels were significantly higher in the thymoquinone treatment groups than those in the model control group. The results of one-way ANOVA indicated that the differences were statistically significant $(\mathrm{P}<0.05)$ and dose-dependent. The $t$-test results showed a significant difference between the low-dose and the high-dose treatment groups $(\mathrm{P}<0.05)$. The pharmacological effects of the drug in the high-dose group were more significant than those in the low-dose group.

Table 1. Effects of thymoquinone on liver biochemical indexes of rats with bile duct ligation (means $\pm \mathrm{SD}$ ).

\begin{tabular}{lcccccc}
\hline Group & Dose & Rat $(\mathrm{N})$ & HP $(\mathrm{mg} / \mathrm{g})$ & MDA $(\mathrm{nmol} / \mathrm{g})$ & SOD $(\mathrm{U} / \mathrm{g})$ & GPx $(\mathrm{nmol} / \mathrm{g})$ \\
\hline Normal control & - & 8 & $226.13 \pm 12.56$ & $186.81 \pm 10.05$ & $5.34 \pm 0.82$ & $0.56 \pm 0.09$ \\
Model control & - & 8 & $568.92 \pm 20.18^{\#}$ & $597.57 \pm 22.31^{\#}$ & $2.15 \pm 0.25^{*}$ & $0.25 \pm 0.04^{\#}$ \\
Low-dose thymoquinone & $25 \mathrm{mg} / \mathrm{kg}$ & 8 & $369.58 \pm 18.39^{\Delta}$ & $324.18 \pm 16.4^{\Delta}$ & $3.46 \pm 0.33^{*}$ & $0.46 \pm 0.04^{\Delta}$ \\
High-dose thymoquinone & $50 \mathrm{mg} / \mathrm{kg}$ & 8 & $282.47 \pm 9.59^{\Delta}$ & $269.77 \pm 18.6^{\Delta}$ & $4.12 \pm 0.38^{\Delta}$ & $0.49 \pm 0.06^{\Delta}$ \\
\hline Versus the model control group ${ }^{*} \mathrm{P}<0.05,{ }^{\Delta} \mathrm{P}<0.01 ;$ versus the normal control oroup, \# $\mathrm{P}<0.01$.
\end{tabular}

Versus the model control group, ${ }^{*} \mathrm{P}<0.05,{ }^{\mathrm{A}}<0.01$; versus the normal control group, ${ }^{\sharp} \mathrm{P}<0.01$. 


\section{Histopathology}

Figure 1 shows hematoxylin and eosin staining of the rat livers in the normal control group. The liver lobules had complete structures without fibrosis and inflammatory cell infiltration. The liver tissue structure of rats in the model control group was incomplete, and significant swelling was observed in the liver cells. Karyopyknosis with significant inflammatory infiltration and fibrosis was observed in the rats in the model control group. However, the liver tissue and cell structures of the rats in the thymoquinone treatment groups were relatively intact with no obvious infiltration and fibrosis. The results in the high-dose group were better than those in the low-dose group. The liver fibrosis scores of the model control, low-dose treatment, and high-dose treatment groups were $2.59 \pm 0.38,1.12 \pm 0.17$, and $0.68 \pm 0.11$, respectively. Table 2 shows the effects of different thymoquinone doses on liver tissue injury in rats with bile duct ligation.

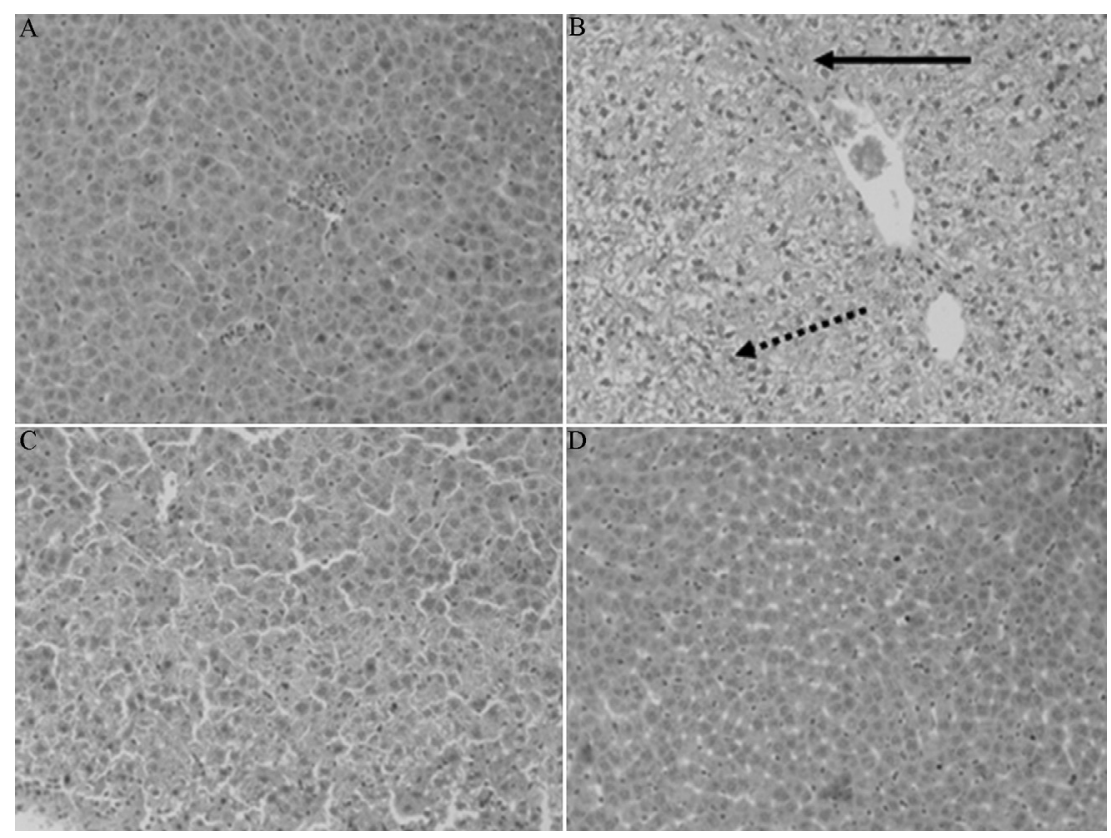

Figure 1. Light microscopy of liver. A. Normal control group. B. Model control group. C. Low-dose thymoquinone group. D. High-dose thymoquinone group. Solid arrow represents fibrosis and inflammatory infiltration. Dotted arrow represents injured hepatocyte.

Table 2. Effects of thymoquinone on liver tissue damage score of rats with bile duct ligation (means $\pm \mathrm{SD}$ ).

\begin{tabular}{lccc}
\hline Group & Dose & Rat (N) & Hepatic histological scoring \\
\hline Normal control & - & 8 & $0.00 \pm 0.00$ \\
Model control & - & 8 & $2.59 \pm 0.38$ \\
Low-dose thymoquinone & $25 \mathrm{mg} \cdot \mathrm{kg}^{-1}$ & 8 & $1.12 \pm 0.17 *$ \\
High-dose thymoquinone & $50 \mathrm{mg} \cdot \mathrm{kg}^{-1}$ & 8 & $0.68 \pm 0.11^{*}$ \\
\hline
\end{tabular}

Versus the model control, $* \mathrm{P}<0.01$. 


\section{DISCUSSION}

The important function of oxidative stress in the pathophysiological process of liver injury and fibrosis induced by biliary occlusion is one of the most studied aspects of cholestatic liver injury treatment (Khorasani et al., 2008; Hayat et al., 2011). Previous studies (LaLonde et al., 1997; Konishi et al., 2006; Nagi et al., 2010) show that model rats with bile duct ligation have pathological features similar to those of human cholestatic liver injury. Biliary occlusion can induce the production of highly active molecules, such as reactive oxygen species and reactive nitrogen radicals, in vivo. Increased oxidative damage occurs and the capacity for scavenging oxygen-free radicals diminishes. Lipid peroxidation and destruction of cell structure induce membrane permeability and cellular metabolic imbalance. Epithelial cell proliferation of the bile duct is promoted. Hepatic fiber scaffold remodeling is induced by activation of the hepatic stellate cells. Such dramatic changes in the cells and tissues contribute to the development of liver fibrosis. Oxidative damage to the biliary pathological process is observed in cholestatic liver injury and in most cases of liver cell injury (Kainuma et al., 2006).

In the present study, the pathophysiological process of human cholestatic liver injury was simulated by artificially establishing bile duct ligation rat models. The protective effects of thymoquinone on liver cell injury induced by bile duct occlusion was investigated further by detecting HP, MDA, SOD, and GPx activities in liver tissues and by liver pathology scoring. The levels of oxidative damage indexes (i.e., HP and MDA) among the groups were in the following order: model control group $>$ low-dose thymoquinone group $>$ high-dose thymoquinone group $>$ normal control group. The levels of the indicators of antioxidative damage (i.e., SOD and GPx) among the groups were in the following order: normal control $>$ high-dose thymoquinone group $>$ low-dose thymoquinone group $>$ model control group. In the bile duct occlusion rat model, the oxidative damage was activated, and the lack of antioxidant capacity increased the liver fibrosis score and the degree of fibrosis. Thymoquinone can significantly reduce oxidative damage, improve the antioxidative effect on the liver, and reduce the fibrosis score (Birkner et al., 2007; Thong-Ngam et al., 2007; Singer et al., 2011). Therefore, we hypothesized that the protective effect of thymoquinone on liver injury was associated with the reduction of hepatic oxidative stress byproduct levels and improvement of the endogenous antioxidant system (Badary et al., 1998; Mansour et al., 2002). The histopathological results showed that thymoquinone could significantly reduce the liver cell damage induced by bile duct ligation and the degree of inflammatory infiltration and fibrosis.

To account for the loss in the absorption of oral thymoquinone, a slightly higher dose of the drug was selected, and two groups administered with high and low doses were observed. The results demonstrated the protective and antioxidant effects of thymoquinone on the hepatocytes in the bile duct occlusion model. The effects of thymoquinone on the reduction of oxidative damage and improvement of hepatic function increased with increasing dose. No obvious liver toxicity was observed at a dose of $50 \mathrm{mg} / \mathrm{kg}$.

In conclusion, the study suggests that thymoquinone has protective effects against oxidative damage and liver fibrosis during the development of extrahepatic cholestasis caused by bile duct ligation. The underlying mechanism is related to body HP content, which reduces and maintains the balance of the oxidative-antioxidative system. This effect participates in liver function protection in patients with cholestasis. 


\title{
Conflicts of interest
}

\author{
The authors declare no conflict of interest.
}

\section{REFERENCES}

Badary O, Al-Shabanah O, Nagi M, Al-Bekairi AM, et al. (1998). Acute and subchronic toxicity of thymoquinone in mice. Drug Dev. Res. 44: 56-61.

Birkner E, Zalejska-Fiolka J, Kasperczyk A, Kasperczyk S, et al. (2007). The influence of methionine, selenomethionine, and vitamin E on liver metabolic pathways and steatosis in high-cholesterol fed rabbits. Biol. Trace Elem. Res. 120: 179-194.

Butler KL, Goverman J, Ma H, Fischman A, et al. (2010). Stem cells and burns: review and therapeutic implications. J. Burn Care Res. 31: 874-881.

Copple BL, Jaeschke H and Klaassen CD (2010). Oxidative stress and the pathogenesis of cholestasis. Semin. Liver. Dis. 30: 195-204.

El-Mahmoudy A, Matsuyama H, Borgan MA, Shimizu Y, et al. (2002). Thymoquinone suppresses expression of inducible nitric oxide synthase in rat macrophages. Int. Immunopharmacol. 2: 1603-1611.

Figiel SC, Franco A, Pucar D, Lewis KN, et al. (2012). Paucity of biliary ducts: A rare etiology of neonatal cholestasis. $J$. Radiol. Case Rep. 6: 29-38.

Galicia-Moreno M, Favari L and Muriel P (2012). Antifibrotic and antioxidant effects of N-acetylcysteine in an experimental cholestatic model. Eur. J. Gastroenterol. Hepatol. 24: 179-185.

Hayat K, Asim MB, Nawaz M, Li M, et al. (2011). Ameliorative effect of thymoquinone on ovalbumin-induced allergic conjunctivitis in Balb/c mice. Curr. Eye Res. 36: 591-598.

Kainuma M, Fujimoto M, Sekiya N, Tsuneyama K, et al. (2006). Cholesterol-fed rabbit as a unique model of nonalcoholic, nonobese, non-insulin-resistant fatty liver disease with characteristic fibrosis. J. Gastroenterol. 41: 971-980.

Kanter M, Demir H, Karakaya C and Ozbek H (2005). Gastroprotective activity of Nigella sativa L oil and its constituent, thymoquinone against acute alcohol-induced gastric mucosal injury in rats. World J. Gastroenterol. 11: 6662-6666.

Khorasani G, Hosseinimehr SJ, Zamani P, Ghasemi M, et al. (2008). The effect of saffron (Crocus sativus) extract for healing of second-degree burn wounds in rats. Keio J. Med. 57: 190-195.

Konishi M, Iwasa M, Araki J, Kobayashi Y, et al. (2006). Increased lipid peroxidation in patients with non-alcoholic fatty liver disease and chronic hepatitis $\mathrm{C}$ as measured by the plasma level of 8-isoprostane. J. Gastroenterol. Hepatol. 21: $1821-1825$.

LaLonde C, Nayak U, Hennigan J and Demling RH (1997). Excessive liver oxidant stress causes mortality in response to burn injury combined with endotoxin and is prevented with antioxidants. J. Burn Care Rehabil. 18: 187-192.

Mansour MA (2000). Protective effects of thymoquinone and desferrioxamine against hepatotoxicity of carbon tetrachloride in mice. Life Sci. 66: 2583-2591.

Mansour MA, Nagi MN, El-Khatib AS and Al-Bekairi AM (2002). Effects of thymoquinone on antioxidant enzyme activities, lipid peroxidation and DT-diaphorase in different tissues of mice: a possible mechanism of action. Cell Biochem. Funct. 20: 143-151.

Nagi MN, Almakki HA, Sayed-Ahmed MM and Al-Bekairi AM (2010). Thymoquinone supplementation reverses acetaminophen-induced oxidative stress, nitric oxide production and energy decline in mice liver. Food Chem. Toxicol. 48: 2361-2365.

Singer AJ, Taira BR, Lin F, Lim T, et al. (2011). Curcumin reduces injury progression in a rat comb burn model. J. Burn Care Res. 32: 135-142.

Thong-Ngam D, Samuhasaneeto S, Kulaputana O and Klaikeaw N (2007). N-acetylcysteine attenuates oxidative stress and liver pathology in rats with non-alcoholic steatohepatitis. World J. Gastroenterol. 13: 5127-5132.

Tomur A, Kanter M, Gurel A and Erboga M (2011). The efficiency of CAPE on retardation of hepatic fibrosis in biliary obstructed rats. J. Mol. Histol. 42: 451-458. 